\title{
MicroRNA 362-3p Reduces hERG-related Current and Inhibits Breast Cancer Cells Proliferation
}

\author{
ABDULLAH A. ASSIRI ${ }^{1,2}$, NOHA MOURAD ${ }^{1,3}$, MINGHAI SHAO ${ }^{1}$, PATRICK KIEL ${ }^{4}$, \\ WANQING LIU ${ }^{5}$, TODD C. SKAAR ${ }^{4}$ and BRIAN R. OVERHOLSER ${ }^{1,4}$ \\ ${ }^{1}$ Department of Pharmacy Practice, College of Pharmacy, Purdue University, West Lafayette, IN, U.S.A.; \\ ${ }^{2}$ Department of Clinical Pharmacy, King Khalid University, Abha, Kingdom of Saudi Arabia; \\ ${ }^{3}$ College of Pharmacy, Manchester University, Fort Wayne, IN, U.S.A.; \\ ${ }^{4}$ Division of Clinical Pharmacology, Indiana University School of Medicine, Indianapolis, IN, U.S.A.; \\ ${ }^{5}$ Department of Pharmaceutical Sciences, Eugene Applebaum College of Pharmacy and Health Sciences, \\ Wayne State University, Detroit, MI, U.S.A.
}

\begin{abstract}
Background/Aim: hERG potassium channels enhance tumor invasiveness and breast cancer proliferation. MicroRNA (miRNA) dysregulation during cancer controls gene regulation. The objective of this study was to identify miRNAs that regulate hERG expression in breast cancer. Materials and Methods: Putative miRNAs targeting hERG were identified by bioinformatic approaches and screened using a 3'UTR luciferase assay. Functional assessments of endogenous $h E R G$ regulation were made using whole-cell electrophysiology, proliferation assays, and cell-cycle analyses following miRNA, hERG siRNA, or control transfection. Results: miR-362-3p targeted hERG 3'UTR and was associated with higher survival rates in patients with breast cancer $(H R=0.39,95 \% C I=0.18$ 0.82). Enhanced miR-362-3p expression reduced $h E R G$ expression, peak current, and cell proliferation in cultured breast cancer cells $(p<0.05)$. Conclusion: miR-362-3p mediates the transcriptional regulation of $h E R G$ and is associated with survival in breast cancer. The potential for miR-362-3p to serve as a biomarker and inform therapeutic strategies warrants further investigation.
\end{abstract}

The human ether-a-go-go-related gene (hERG, KCNH2) codes for rectifier voltage-gated potassium channels (Kv11.1) that are vital to diverse physiological processes.

This article is freely accessible online.

Correspondence to: Brian R. Overholser, Ph.D., FCCP, Purdue University College of Pharmacy, Research Institute 2, Room 402, 950 W. Walnut St., Indianapolis, IN 46202, U.S.A. Tel: +1 3172784001, e-mail: boverhol@purdue.edu

Key Words: MicroRNA, hERG, breast cancer, miR-362-3p, voltagegated potassium channels.
Most notably, these voltage-gated potassium channels are essential in cardiac tissue as they regulate the chief repolarizing current $\mathrm{I}_{\mathrm{Kr}}$ (1). However, in addition to regulating repolarization in cardiac cells, these channels are important in other critical biological processes (2). For example, hERG potassium channels are expressed in smooth muscles and endocrine cells (2). The physiological importance of hERG is multifaceted as reflected by a variety of roles based on cell or tissue types. In addition to the well characterized and important physiological roles, the hERG channel has been implicated as a potential oncogene (3-5).

The hERG protein is overexpressed in several types of cancer, such as, breast cancer, glioblastoma, and colorectal cancer, even when the originating tissue does not express the potassium channel (5-15). The expression of these channels has been reported to contribute to the pathological regulation of malignancies by facilitating cell proliferation, differentiation and regulating apoptosis (16). The pathological consequences of hERG overexpression has been implicated in maintaining a repolarized membrane potential that facilitates progression of the cell cycle and accelerates the growth and invasiveness of tumor (16). Inhibition of hERG channels has been reported to promote apoptosis and decrease invasion in glioblastoma and other cancer cells by arresting cells in the $G_{0} / G_{1}$ phase of the cell cycle $(3,8)$. Thus, the aberrant expression of voltage-gated potassium channels is involved in cell growth and apoptosis regulation in non-excitable cells, such as tumor cells (17).

The transcriptional regulation of hERG channels in cancer is largely unknown but is likely due to the common epigenetic alterations in tumor cells. MicroRNAs (miRNAs) are endogenous, non-protein coding RNAs that control gene expression by pairing to the 3'UTR region of target transcripts to silence gene expression by mRNA degradation or repressing protein expression $(18,19)$. They regulate multiple cellular processes in cancer including cell 
proliferation, cell invasion and metastasis. In fact, miR-96 and miR-493 have been shown to target hERG resulting in decreased proliferation, migration and invasion of pancreatic cancer $(20,21)$. In fact, the roles of miRNAs have been demonstrated in several types of cancer, including breast cancer (22).

Given the potential importance of hERG expression in certain cancers and the fact that gene expression is regulated by miRNAs, our primary objective was to screen, identify, and explore the effects of miRNAs that potentially target and regulate hERG expression in breast cancer. We utilized bioinformatic and molecular approaches to examine the role of miRNAs in the regulation of hERG channels expression and function in cultured breast cancer cells. The results of this investigation provide further insights into the understanding of miRNA-hERG regulation in cancer which may inform future work toward diagnostic or prognostic biomarkers.

\section{Materials and Methods}

Bioinformatic approach. MiRNAs were globally screened to identify those that target the hERG gene based on predictions using the miRWalk database (http://zmf.umm.uni-heidelberg.de/apps/ zmf/mirwalk2/). The four default algorithms: miRWalk, RNA22, miRanda, and TargetScan were used to predict putative miRNAs that regulate hERG. Only human data were searched for the gene of interest, $\mathrm{KCNH} 2$ (transcripts 1 and 2 ). The input parameters used the 3'UTR as a target location for putative miRNAs that had a minimum seed length of 7 mers and/or $p$-value of 0.05 . The predicted miRNAs were further screened through the miRCancer database (www.mircancer.ecu.edu), which provides a comprehensive collection of miRNAs-hERG expression profile, and their role in human breast cancer (23). The utilization of both the miRWalk database and the miRCancer database were chosen to improve prediction accuracy and reduce false positive prediction. The overlapped miRNAs identified by the prediction algorithms, with reported benefit in human breast cancer as shown in the miRCancer database, were used for subsequent analysis. OncoLnc (http://www.oncolnc.org) was used to explore survival correlations related to the miRNAs identified from the bioinformatics approach. The OncoLnc dataset contains survival data for 198 patients from breast invasive carcinoma (BRCA) studies performed by The Cancer Genome Atlas (TCGA).

Cell culture and transfection. Human breast cancer cell lines (SKBR-3, MCF-7) were grown in McCoy's 5A medium (ATCC ${ }^{\circledR} 30-$ 2020TM), or EMEM medium (ATCC ${ }^{\circledR} 30-2003^{\mathrm{TM}}$ ) containing $10 \%$ (v/v) Fetal Bovine Serum (FBS; ATCC ${ }^{\circledR} 30-2020^{\mathrm{TM}}$ ) and penicillin/streptomycin $1 \%(\mathrm{v} / \mathrm{v})$. SK-BR-3 is a TP53 mutated invasive ductal carcinoma cell line which was derived from a pleural effusion and known to overexpress the HER2 gene. MCF-7 is an invasive ductal carcinoma cell line that expresses estrogen receptors. Both cell lines endogenously express the $h E R G$ gene (16). The cells were maintained at $37^{\circ} \mathrm{C}$ in an atmosphere containing $5 \%$ $\mathrm{CO}_{2}$. The negative control miRNAs, MISSION miRNAs mimics, and siRNAs were purchased from Sigma Aldrich (St. Louis, MO, USA) and Santa Cruz Biotechnology (Dallas, TX, USA). Transfection and co-transfection were performed using lipofectamine 2000 (Thermo Fisher Scientific ${ }^{\circledR}$, Waltham, MA, USA) according to manufacturer's protocol.
Luciferase assay. The miRNAs identified through the bioinformatic approach were screened using a Dual-Luciferase Assay (Promega, Madison, WI, USA) to quantify hERG 3'-UTR activity. The hERG 3'-UTR reporter clone (pLenti-UTR-LUC) was purchased from Applied Biological Materials (Richmond, BC, Canada). SK-BR-3 cells were co-transfected with MISSION miRNA mimics or negative control with the pLenti-UTR-LUC and control reporter plasmid (renilla) using Lipofectamine 2000 (Thermo Fisher Scientific). Luminescence was measured using a TD-20/20 Luminometer. Relative luciferase activity was normalized to renilla to control for transfection efficiency. Experiments were performed in triplicate and repeated in independent conditions.

Quantitative reverse transcriptase-polymerase chain reaction ( $q R T$ PCR) analysis. A total of $500 \mathrm{ng}$ RNA of each sample was reverse transcribed using the High Capacity RNA-to-cDNA Kit (Applied Biosystems, Foster City, CA, USA) according to manufacturer's protocol. The extracted RNA was mixed with RT Buffer, enzyme mix, random sequence oligonucleotide primers, and nuclease-free water and cDNA was synthesized. Specific primers targeting either hERG mRNA or the endogenous control, GAPDH, were purchased from Applied Biosystems (Taqman ${ }^{\circledR}$ assays, Foster City, CA, USA). The TaqMan ${ }^{\circledR}$ Gene Expression Kit (Life Technology, Carlsbad, CA, USA) and QuantStudio 12K Flex Real-Time PCR system (Applied Biosystems, Foster City, CA, USA) was used to quantify the level of expression of hERG mRNA in cell lines transfected with miRNAs mimics or negative control (Sigma Aldrich). The

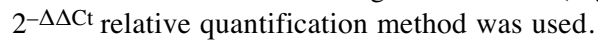

hERG protein immunoblot. Cells were washed with phosphatebuffered salt solution (PBS; Thermo Fisher Scientific) and then lysed (RIPA, Beyotime, Shanghai, PR China). The protein concentration was quantified using the bicinchoninic acid assay. A uniform $5 \mu \mathrm{g}$ of total protein was loaded per lane and samples were fractionated on 6-12\% sodium dodecyl sulfate-polyacrylamide gradient gel (Novex ${ }^{\circledR}$ Bis-Tris Bolt ${ }^{\circledR}$ SDS-PAGE gel, Thermo Fisher Scientific), transferred to nitrocellulose membranes (Thermo Fisher Scientific) and blocked with $5 \%$ bovine serum albumin (BSA, Sigma Aldrich). The membrane was exposed to anit-hERG primary antibodies (Cell Signaling, Danvers, MA, USA), and anti-GAPDH (Santa Cruz Biotechnology, Dallas, TX, USA) overnight at $4^{\circ} \mathrm{C}$. Following a wash, the membrane was probed with secondary antibody (Santa Cruz Biotechnology) and developed using Pierce ECL Western Blotting Substrate (Thermo Fisher Scientific). Images were acquired from the Li-Cor Odyssey imaging system and analyzed with Image ${ }^{\circledR}$ Software.

Electrophysiology. Cells were co-transfected with a pcDNA3.1 vector containing GFP and MISSION hsa-miR-362-3p using Lipofectamine 2000. Functional measurements of hERG-related current were assessed between 24- and 48-h following transfection using the whole cell, patch-clamp configuration in the voltage clamp mode. Recordings were performed at room temperature $\left(\sim 22^{\circ} \mathrm{C}\right)$ and data were acquired using an EPC-9 amplifier and Patchmaster software (HEKA Instruments Inc, Holliston, MA, USA). Borosilicate glass micropipettes were pulled using a P-2000 Puller (Sutter Instrument, Novato, CA, USA) to a resistance of 2-8 M $\Omega$. Micropipettes were filled with pipette solution, containing (in $\mathrm{mM}$ ): $130 \mathrm{KCl}, 1 \mathrm{MgCl}_{2}$, 5 EGTA, $5 \mathrm{MgATP}$, and 10 HEPES The external solution contained (in $\mathrm{mM}$ ): $137 \mathrm{NaCl}, 4 \mathrm{KCl}, 1.8 \mathrm{CaCl}_{2}, 1 \mathrm{MgCl}_{2}$, 
Table I. Bioinformatics approaches to identify miRNAs predicted to bind to hERG with known beneficial effect in breast cancer.

miRWALK database

(http://zmf.umm.uni-heidelberg.de/apps/zmf/mirwalk2/)

Prediction algorithms

Putative miRNAs-hERG identified by each algorithm

miRCancer database

hERG-putative miRNAs associated with breast cancer benefits (miRCancer database)

miRNAs identified by at least 2 algorithms

$\begin{array}{cccc}\text { miRWALK } & \text { miRANDA } & \text { RNA22 } & \text { Targetscan } \\ 432 \text { miRNAs } & 266 \text { miRNAs } & 436 \text { miRNAs } & 375 \text { miRNAs }\end{array}$

256 miRNAs associated with benefit in human breast cancer

miR-494-3p miR-362-3p miR-625-5p miR-362-3p miR-497-5p miR-199b-3p miR-625-5p miR-625-5p
10 HEPES, and 10 glucose. External solution was adjusted to a $\mathrm{pH}$ of 7.4 using $\mathrm{NaOH}$, and pipette solution was adjusted to a $\mathrm{pH}$ of 7.2 with KOH. hERG tail currents were elicited in SK-BR-3 cells using the following voltage protocol: from a holding potential of $-40 \mathrm{mV}$, hERG channels were activated using a $2.5 \mathrm{sec}$ depolarizing step to $+40 \mathrm{mV}$ followed by a series of $100 \mathrm{msec}$ repolarizing test pulses between $-120 \mathrm{mV}$ and $+30 \mathrm{mV}$ to elicit tail currents. All electrophysiology data were analyzed using FitMaster v2x73.1.

Cell proliferation assay. Cell proliferation was detected using the CellTiter Aqueous One Solution Cell Proliferation Assay (MTS, Promega). The miR-362-3p mimic and negative control (Sigma Aldrich) were transfected into cultured cancer cells, harvested, and seeded in 96-well plates (Coring Incorporated ${ }^{\circledR}$, Corning, NY, USA). Cells were dissociated into single-cell suspensions by digestion with $0.9 \%$ trypsin at $37^{\circ} \mathrm{C}$. Cell proliferation was examined after $48 \mathrm{~h}$ in $100 \mu \mathrm{l}$ of culture medium and $20 \mu \mathrm{l}$ of CellTiter Aqueous One Solution. Following a 4-h incubation at $37^{\circ} \mathrm{C}$, the absorbance was measured at a wavelength of $490 \mathrm{~nm}$ (with $630 \mathrm{~nm}$ as the reference wavelength) using an ELISA microplate reader (Shenzhen Highcreation Technology $\mathrm{Co}^{\circledR}$, Turku, Finland). All proliferation assays were repeated in triplicate.

Cell cycle analysis. Flow cytometry was used to differentiate phases of the cell cycle following transfection with miR-362-3p, negative control, or hERG siRNA (Sigma Aldrich). Cells were trypsinized 48 $\mathrm{h}$ following-transfection, fixed and permeabilized using $70 \%$ ethanol, rinsed in phosphate-buffered saline (PBS, Thermo Fisher Scientific), and treated with RNase A. DNA was stained with propidium iodide for $30 \mathrm{~min}$ at room temperature and protected from light. Fluorescence was analyzed using a BD LSRFORTESSA flow cytometer and ModFit LT software.

Statistical analysis. Data were compared via one-way ANOVA with Dunnett's test post-hoc test or independent sample t-test, as appropriate, using GraphPad Prism v7.4 (GraphPad Software, San Diego, CA, USA). The associations between miRNA expression and days to all-cause mortality were analyzed via a Kaplan-Meier survival analysis with the $p$-value calculated by the log-rank test. For all statistical comparisons, $\alpha$ was set to 0.05 .

\section{Results}

microRNAs predicted to target hERG channels. The number of miRNAs predicted to bind to hERG by the four prediction algorithms were as follows; 432 by miRWalk, 266 by miRanda, 436 by RNA22, and 432 by TargetScan. To minimize false positive predictions, the miRCancer database was used to search for dysregulated miRNAs that have been previously associated with beneficial effects in breast cancer. The miRCancer online database reported 256 dysregulated miRNAs in breast cancer. Among the 256 miRNAs, five miRNAs (miR-199b-5p, miR-362-3p, miR-494, miR-497 and miR-625-5p) were predicted by at least one of the four prediction algorithms to regulate the hERG 3'UTR, as displayed in Table I. Two putative miRNAs (miR-362-3p \& miR-625-5p) were predicted to bind hERG by at least two of the prediction algorithms.

Lower expression of miR-362-3p is associated with longer survival in breast cancer. Kaplan-Meier survival analyses were performed to examine the strength of association of the expression of the five putative miRNAs (miR-199b-5p, miR362-3p, miR-494, miR-497 and miR-625-5p) with clinical outcomes using The TCGA survival data with miRNA expression data using OncoLnc online tool. To examine the influence of miRNAs on survival, the survival rate between patients with the highest expression of miRNAs (90th percentile; $n=98$ ) was compared with patients with the lowest expression (10th percentile; $n=98$ ). The survival rate in patients with low miR-362-3p expression (10th percentile) was significantly reduced when compared with patients with high miR-362-3p expression (90th percentile) by log-rank test $(\mathrm{HR}=0.39,95 \% \mathrm{CI}=0.18-0.82, p=0.012)$ as displayed in Figure 1 . There was not a statistically significant difference in overall survival between patients with low (10th percentile) and 

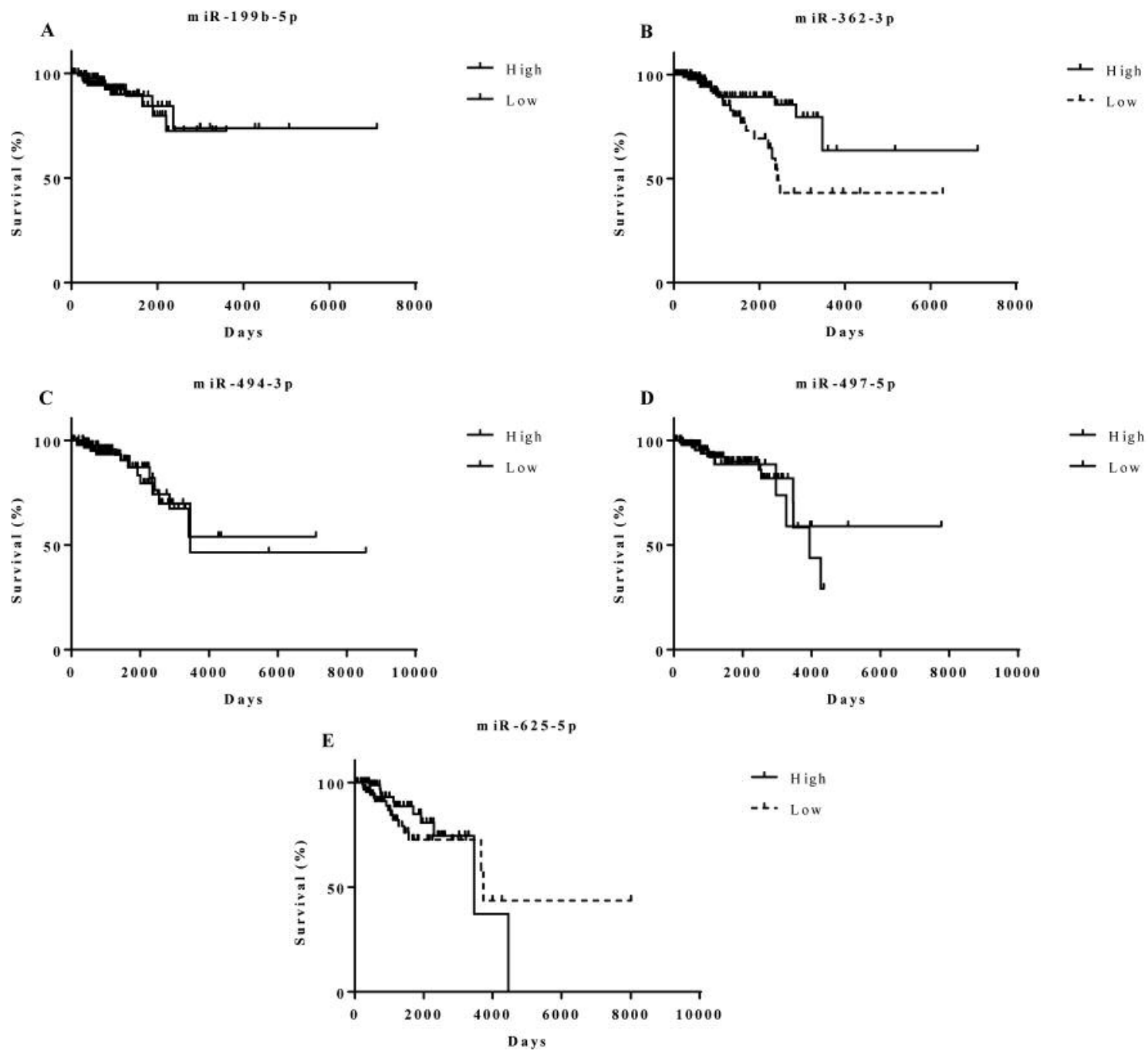

$$
\begin{aligned}
& \text { - High } \\
& \text {-L. Low }
\end{aligned}
$$

Figure 1. Kaplan-Meier curves from The TCGA in breast cancer patients with the 10th (Low) and 90th (High) percentile of miRNA expression levels (fragments per kilobase million): (A) miR-199b-5p high expression patients (647 \pm 184$)$ versus miR-199b-5p low expression patients (49.6 \pm 17.9$)$. (B) miR-362-3p high expression patients (5.9 \pm 2.8$)$ versus miR-362-3p low expression patients $(0.01 \pm 0.05) ; p=0.012$ (HR=0.39, 95\%CI=0.18-0.82). (C) miR-494-3p high expression patients (6.2 \pm 3.9$)$ versus miR-494-3p low expression patients $(0.0 \pm 0.0)$. (D) miR-497-5p high expression patients (73.2 \pm 24.3$)$ versus miR-497-5p low expression patients $(6.5 \pm 1.7)$. (E) miR-625-5p high expression patients (12.3 \pm 5.2$)$ versus miR-625-5p low expression patients $(0.5 \pm 0.3)$.

patients with high expression (90th percentile) with the other four miRNAs; including miR-625-5p expression which was the other miRNA predicted to regulate hERG by more than one algorithm $(\mathrm{HR}=0.93,95 \% \mathrm{CI}=0.43-2, p=0.36)$.

miR-362-3p decreases hERG 3'UTR activity. The five putative miRNAs (miR-199b-5p, miR-362-3p, miR-494, miR-497 and miR-625-5p) were screened for hERG 3'-UTR activity using a Luciferase assay. miR-362-3p significantly decreased luciferase activity by $10 \% \pm 2.3(p<0.001)$ when compared to cells transfected with the control plasmid. The four other miRNAs, including miR-625-5p $(0.66 \% \pm 3.56$; $p=0.55)$, did not significantly reduce luciferase activity, as displayed in Figure 2A.

miR-362-3p decreases hERG expression in breast cancer cells. Based on the bioinformatics approach, survival analysis, and luciferase screening, miR-362-3p was screened further for potential hERG regulation. miR-362-3p and siRNA hERG (positive control) significantly decreased hERG mRNA expression by $15.9 \% \pm 6.0(p<0.001, \mathrm{n}=3)$, and $42.3 \% \pm 4.0$ $(p=0.01, \mathrm{n}=3)$, respectively in SK-BR-3 cells. Similarly in MCF-7, miR-362-3p and siRNA hERG significantly decreased $\mathrm{hERG}$ mRNA expression by $25.7 \% \pm 11.1(p=0.02$, 
A

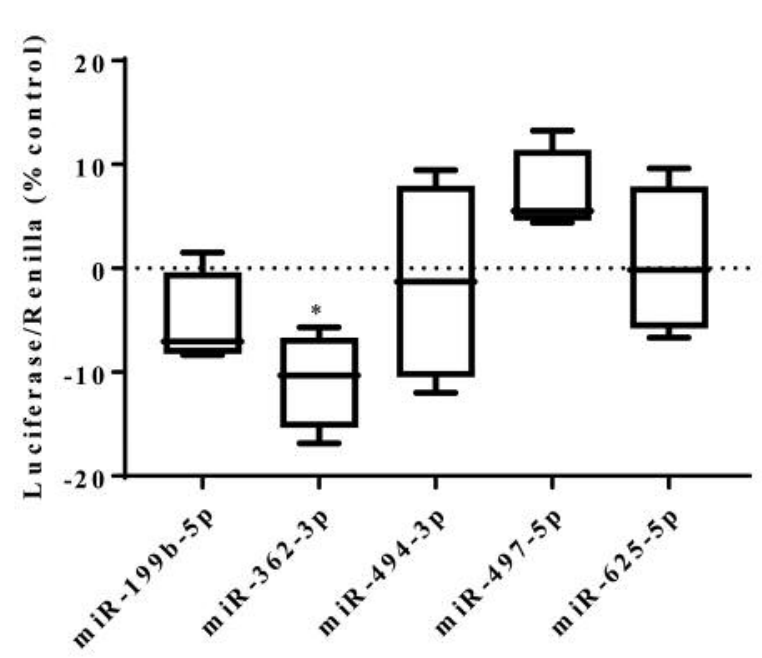

B

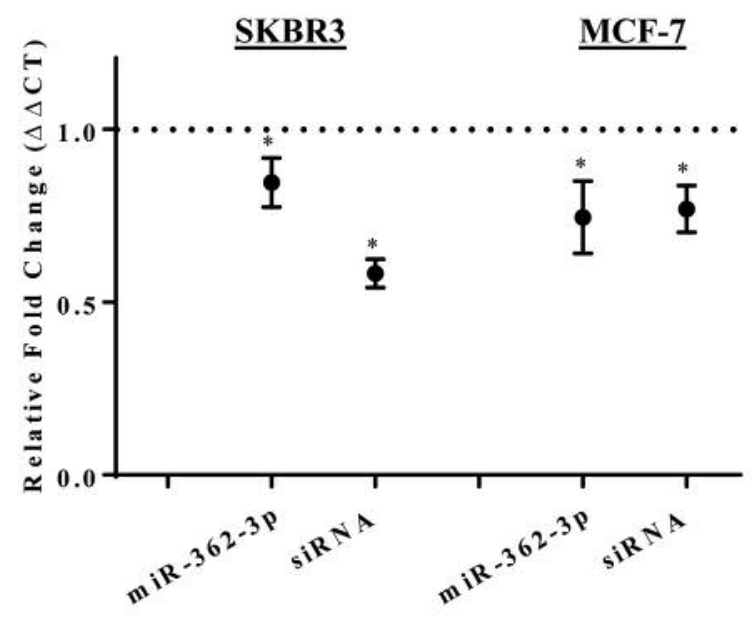

Figure 2. miR-362-3p reduces hERG 3'UTR activity and mRNA expression. (A) Luciferase hERG 3'UTR screening of the five putative miRNAs. Only miR-362-3p significantly reduced hERG 3'UTR activity with a reduction of $10 \% \pm 2.3(p<0.001)$. (B) $h E R G$ mRNA expression in SK-BR-3 and $M C F-7$ cells following miR-362-3p mimic or hERG siRNA transfection. Both miR-362-3p and siRNA significantly decreased hERG $m R N A$ in SK$B R-3$ and MCF-7 cells. Experiments were performed as triplicates with error bars depicting standard error of mean. Asterisks denote $p<0.05$.

$\mathrm{n}=3)$, and $23.7 \% \pm 6.4(p=0.03, \mathrm{n}=3)$, respectively as displayed in Figure 2B.

Effect of miR-362-3p on hERG protein expression and function. miR-362-3p decreased immature endogenous hERG $(0.18 \pm 0.03$ to $0.08 \pm 0.01, p=0.038, \mathrm{n}=4)$ by an average of $47 \%$ relative to GAPDH, and mature (i.e. fully glycosylated) endogenous hERG $(0.16 \pm 0.03$ to $0.08 \pm 0.01, p=0.0369, \mathrm{n}=4)$ by an average of $51 \%$ relative to GAPDH, as shown in Figure $3 \mathrm{~A}$ and $\mathrm{B}$. To assess the effect of miR-362-3p on hERGrelated current in SK-BR-3 cells, MISSION miRNA mimic hsa-miR-362-3p was co-transfected with a pcDNA3.1 vector containing GFP. Peak inward hERG-related current tail currents recorded during the repolarizing step to $-120 \mathrm{mV}$ were compared between control and miR-362-3p-transfected cells. miR 362-3p decreased inward peak hERG-related current by an average (SEM) of $52.6 \pm 12.8 \%$ versus control $(\mathrm{n}=6$ per group; $p<0.01)$. Figure $3 \mathrm{C}$ and $\mathrm{D}$ display the absolute reduced peak hERG-related current $(\mathrm{pA} / \mathrm{pF})$ in cells transfected with miR-362-3p.

miR-362-3p decreases cells proliferation in breast cancer cell lines. The role of miR-362-3p in cell proliferation was assessed with MTS assays in both SK-BR-3 and MCF-7 cancer cells. The assay was performed $48 \mathrm{~h}$ following transfection with miR-362-3p mimic or negative control. As displayed in Figure 4 , miR-362-3p significantly decreased SK-BR-3 cells by $23 \% \pm 8.68(p=0.014, \mathrm{n}=3)$ when compared with control wells.
A similar effect of miR-362-3p was observed in MCF-7 cells where miR-362-3p transfected cells were $11.7 \% \pm 1.0(p<0.001$, $\mathrm{n}=3$ ) lower than the control group at $48 \mathrm{~h}$ (Figure 4).

miR-362-3p and siRNA hERG significantly increased the accumulation of cells count in $G_{0} / G_{1}$ phase in MCF-7 breast cancer cells. To elucidate the effect of miR-362-3p on the cell cycle, the effects of miR-362-3p on SK-BR-3 and MCF-7 cells were assessed. miR-362-3p did not significantly increase the accumulation of cells in G0/G1 phase in SK-BR-3 cells with an average increase of $5.8 \%$ (from $59.6 \% \pm 0.12$ to $63.0 \pm 0.74$, $p=0.06, \mathrm{n}=3$ ) when compared to control group. However, siRNA hERG unexpectedly decreased the accumulation of cells in G0/G1 phase in SK-BR-3 cells by $5.13 \%$ (from $59.6 \% \pm 0.12$ to $56.3 \pm 0.73, p=0.006, \mathrm{n}=3$ ) as displayed in Figure $5 \mathrm{~A}$ and $\mathrm{B}$. On the other hand, miR-362-3p significantly increased the accumulation of cells in G0/G1 phase in MCF-7 cells by $11.7 \%$ (from $51.1 \% \pm 0.64$ to $57.1 \pm 0.96, p=0.002, \mathrm{n}=3$ ) when compared to control (Figure 5C and D). Additionally, siRNA hERG demonstrated similar effects to miR-362-3p in MCF-7 cells, in which siRNA hERG increased cells count in G0/G1 phase by $10 \%$ (from $51.1 \% \pm 0.64$ to $56.8 \pm 0.96, p<0.001, \mathrm{n}=3$ ).

\section{Discussion}

Breast cancer is the most common cancer among females worldwide and the second leading cause of all cancer related deaths (24). The hERG gene is expressed in certain cancers 
A

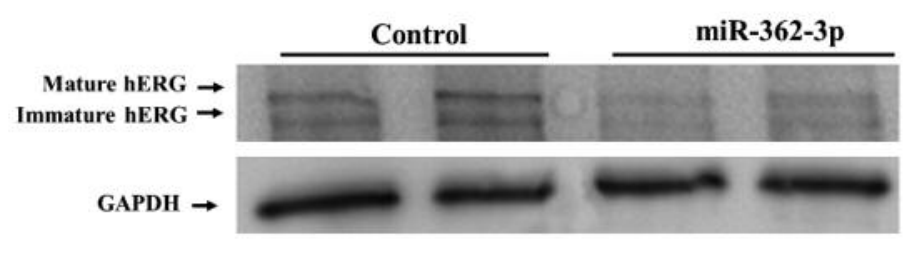

C
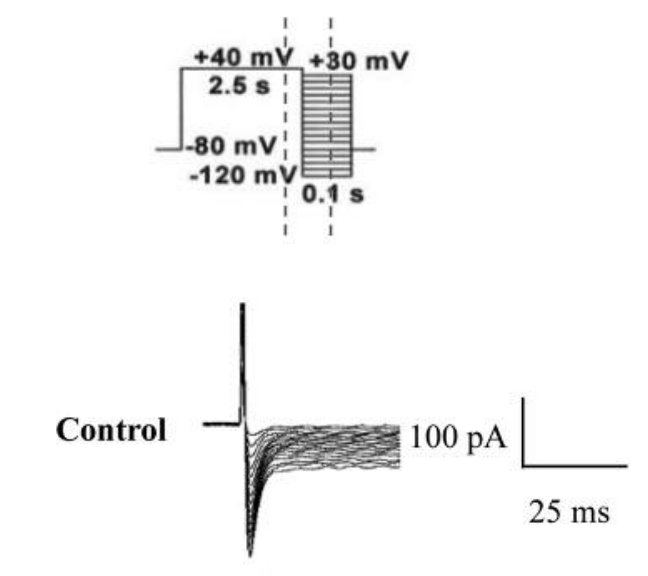

miR-362-3p
B

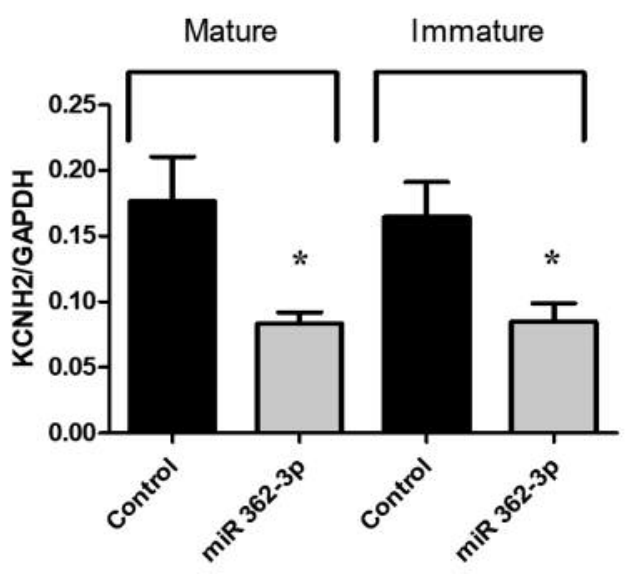

D

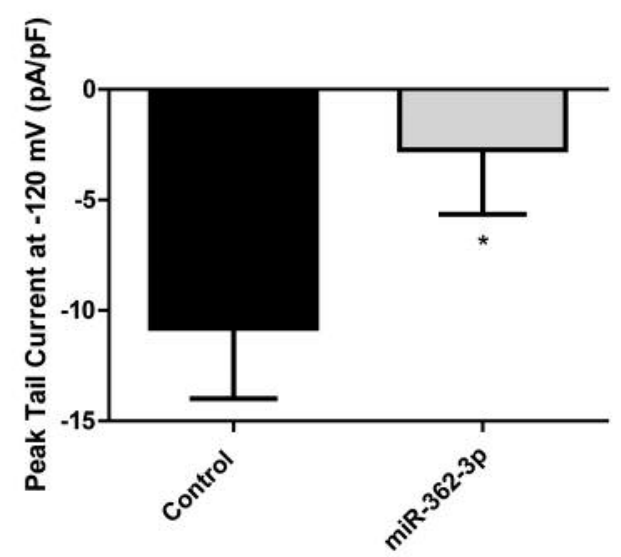

Figure 3. miR-362-3p reduces hERG protein expression and tail current density. (A) Representative protein immunoblots of mature and immature hERG following miR-362-3p or negative control transfection in SK-BR-3 cells. (B) Mature and immature hERG protein expression versus negative control was decreased following miR-362-3p expression in $S K-B R-3$. Experiments were performed as triplicates with error bars depicting standard error of mean. Asterisks denote significance. (C) Endogenous hERG-related tail current was elicited in SK-BR-3 cells using the displayed voltagestep protocol. (D) Tail current density decreased following miR-362-3p transfection versus control ( $n=6$ per group) at the maximum current density elicited at $-120 \mathrm{mV}$.

and has been associated with cancer development and progression $(2,25)$. Furthermore, inhibition of the hERGrelated potassium channel has been shown to impair cell proliferation and the invasiveness of tumor cells including colorectal cancer, glioma, breast cancer, and leukemias (1114, 26-28). In this study, we identified miR-362-3p as a potential regulator of hERG expression and function in breast cancer cells.

miR-362-3p has been shown to target oncogenes and exert beneficial effects. Expression of miR-362-3p has been investigated in several types of cancer and is negatively correlated with tumor progression in primary colorectal cancer (29-36). MicroRNAs commonly target several genes. Indeed, in addition to hERG in the current study, the tumor suppressors E2F1, USF2 and PTPN1 have been identified as targets of miR362-3p which decrease their expression in colorectal cancer. The overexpression of all three of these target genes in colorectal cancer has been associated with increased proliferation and tumor progression (29). Down-regulation of miR-362-3p has also been shown to promote tumor progression in breast cancer and cervical adenocarcinoma $(30,33)$.

miR-362-3p may have counter-acting effects in certain cancers through targeting of tumor suppressors. In hepatocellular carcinoma, miR-362-3p targets the antiproliferative Tob2 gene and may contribute to malignancy (32). Additionally, $C D 82$ has been identified as a direct 
MTS of miR-362-3p

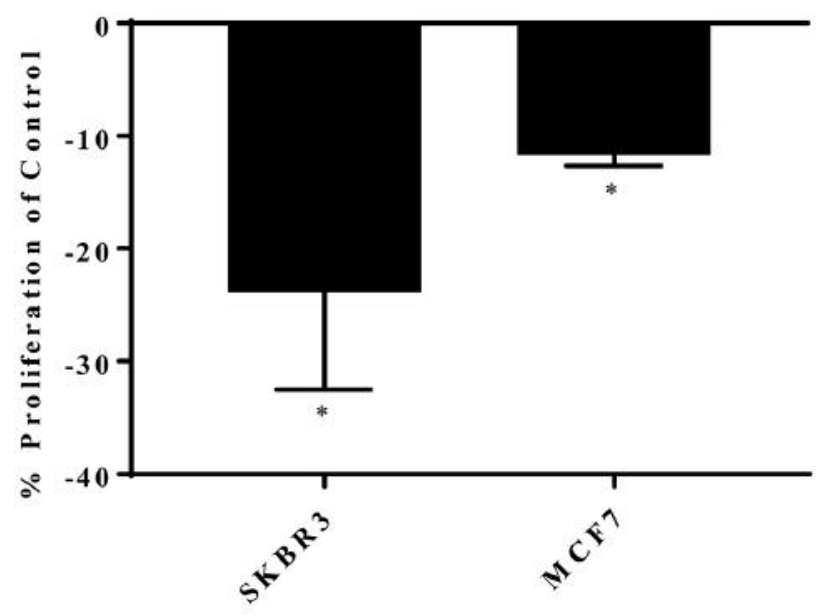

Figure 4. Effect of miR-362-3p on SK-BR-3 and MCF-7 cell proliferation. Cell proliferation was significantly inhibited in miR-362$3 p$ transfected cells versus negative control. Experiments were performed in triplicates with error bars depicting standard error of mean. Asterisks denote $p<0.05$.

target of miR-362-3p. CD82 is a metastasis suppressor known to regulate epithelial-to-mesenchymal cell transition (EMT). In gastric cancer, miR-362-3p expression is higher than in normal gastric mucosa cells (35). Therefore, antimiR-362-3p was demonstrated to inhibit the migration and invasion of gastric cancer (35). Thus, despite the evidence that $\mathrm{miR}-362-3 \mathrm{p}$ expression is associated with improved cancer outcomes, it may also possess counteractive oncogene activity via Tob2 or CD82 targeting.

Pervious work has shown that hERG channel inhibition can reduce cancer cell growth in MCF-7 cells, indicating the oncogenic effect of hERG in these breast cancer cell lines (37). In the current study, miR-362-3p inhibited hERG expression and reduced hERG-related current with antiproliferative effects similar to the hERG-siRNA in breast cancer cells. miR-362-3p has also been shown to decrease p130Cas expression in breast cancer (30). p130Cas overexpression has been associated with poor prognosis in breast cancer. Together, hERG gene might be involved in the downstream effect of miR-362-3p along with p130Cas.

hERG channels have been implicated in different phases of cell cycle progression (38). Therefore, a cell-cycle analysis was performed to the contribution of miR-362-3p in breast cancer progression through hERG regulation. Indeed, breast cancer cells (MCF-7) transfected with miR-362-3p and hERGsiRNA increased accumulation of cells in the G1 phase. These findings are similar to previous studies in glioblastoma which found that hERG inhibitors significantly arrest cancer cells in the G1 phase (38). However, there was no significant change in SK-BR-3 cells when compared with control cells, indicating that any potential beneficial effect of miR-362-3p is likely independent of the cell cycle regulation in SK-BR-3 cells. There are two prevailing explanations for the distinct effects of miR-362-3p and siRNA hERG in MCF-7 and SK-BR-3 cells. First, MCF-7 and SK-BR-3 cells are genetically different; thus, diverse tumorigenic pathways likely drive proliferation and invasion. Secondly, the primary evidence for the hERG gene being involved in cell cycle regulation is from neuroblastoma and colorectal cancer $(29,38)$. The actual involvement of hERG channels in the cell-cycle regulation of breast cancer cells has not been investigated.

In conclusion, our study shows that miR-362-3p regulates hERG expression and function in breast cancer cells. Furthermore, we have shown that overexpression of miR362-3p can inhibit hERG related current and breast cancer proliferation, and that high expression levels of miR-362-3p expression are positively correlated with survival. Based on the multiple downstream targets of miR-362-3p and its positive correlation with survival, a prospective analysis is warranted to assess miR-362-3p expression as a potential prognostic biomarker in breast cancer. Additionally, the precise role of hERG channels in breast cancer, as well as, the ability of miR-362-3p to regulate breast cancer cells growth through targeting hERG channel expression and regulation warrants further investigation.

\section{Conflicts of Interest}

The Authors have no conflicts of interest to declare regarding this study.

\section{Authors' Contributions}

Concept/design: AAA, WL and BRO; Data analysis/interpretation: AAA, NM, WL, and BRO; Drafting article: AAA, NM, and BRO; Critical revision of article: PK, WL, and TCS; Approval of article: AAA, NM, MS, PK, WL, TCS, and BRO; Statistics: AAA, NM, BRO; Funding secured by: BRO; Data collection: AAA, NM, and MS.

\section{Acknowledgements}

Supported by a grant from the American Heart Association (16GRNT31080006). This investigation was also supported in part with support from the Indiana Clinical and Translational Sciences Institute funded, in part, by grant number UL1TR001108 from the National Institutes of Health, National Center for Advancing Translational Sciences, Clinical and Translational Sciences Award.

\section{References}

1 Chiamvimonvat N, Chen-Izu Y, Clancy CE, Deschenes I, Dobrev D, Heijman J, Izu L, Qu Z, Ripplinger CM, Vandenberg JI, Weiss JN, Koren G, Banyasz T, Grandi E, Sanguinetti MC, Bers DM and Nerbonne JM: Potassium currents in the heart: functional roles in repolarization, arrhythmia and therapeutics. J Physiol 595(7): 2229-2252, 2017. PMID: 27808412. DOI: 10.1113/jp272883 

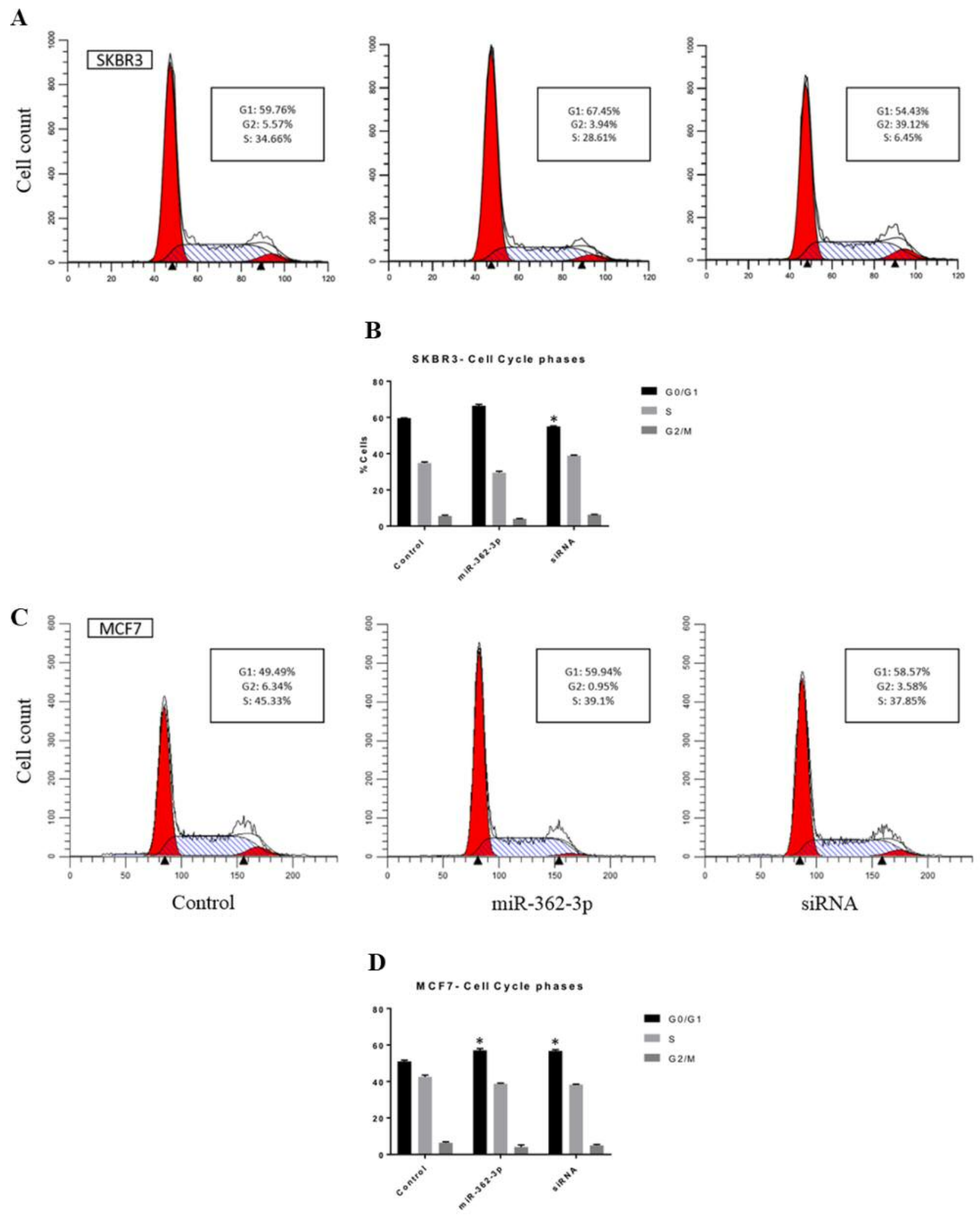

Figure 5. Effect of miR-362-3p and hERG siRNA on cell cycle in breast cancer cells. (A) Representative flow cytometry experiments in SK-Br-3 cells; (B) mean $\pm S E M G_{0} / G_{1}, S$, and $G_{2} / M$ phase in $S K-B r-3$ cells; (C) Representative flow cytometry experiments in MCF-7 cells; $(D)$ mean $\pm S E M G_{0} / G_{1}, S$, and $G_{2} / M$ phases in MCF-7 cells. Experiments were performed in triplicates with error bars depicting standard error of mean. Asterisks denote $p<0.05$. 
2 Babcock JJ and Li M: hERG channel function: beyond long QT. Acta Pharmacol Sin 34(3): 329-335, 2013. PMID: 23459091. DOI: 10.1038/aps.2013.6

3 Arcangeli A: Expression and role of hERG channels in cancer cells. Novartis Foundation Symposium 266: 225-232, 2005. DOI: 10.1002/047002142X.ch 17

4 Arcangeli A and Becchetti A: hERG channels: From antitargets to novel targets for cancer therapy. Clin Cancer Res 23(1): 3 5, 2017. PMID: 27903676. DOI: 10.1158/1078-0432.Ccr-162322

5 Dolderer JH, Schuldes H, Bockhorn H, Altmannsberger M, Lambers C, von Zabern D, Jonas D, Schwegler H, Linke R and Schroder UH: HERG1 gene expression as a specific tumor marker in colorectal tissues. Eur J Surg Oncol 36(1): 72-77, 2010. PMID: 19577877. DOI: 10.1016/j.ejso.2009.05.009

6 Guasti L, Crociani O, Redaelli E, Pillozzi S, Polvani S, Masselli M, Mello T, Galli A, Amedei A, Wymore RS, Wanke E and Arcangeli A: Identification of a posttranslational mechanism for the regulation of hERG1 $\mathrm{K}+$ channel expression and hERG1 current density in tumor cells. Mol Cell Biol 28(16): 5043-5060, 2008. PMID: 18559421 . DOI: $10.1128 / \mathrm{mcb} .00304-087$

7 Patane S: HERG-targeted therapy in both cancer and cardiovascular system with cardiovascular drugs. Int J Cardiol 176(3): 1082-1085, 2014. PMID: 25218820. DOI: 10.1016/ j.ijcard.2014.07.129

8 Afrasiabi E, Hietamaki M, Viitanen T, Sukumaran P, Bergelin N and Tornquist K: Expression and significance of HERG (KCNH2) potassium channels in the regulation of MDA-MB-435S melanoma cell proliferation and migration. Cell Signal 22(1): 57-64, 2010. PMID: 19765650. DOI: 10.1016/j.cellsig.2009.09.010

9 Erdem M, Tekiner TA, Fejzullahu A, Akan G, Anak S, Saribeyoglu ET, Ozbek U and Atalar F: herg1b expression as a potential specific marker in pediatric acute myeloid leukemia patients with HERG 897K/K genotype. Pediatr Hematol Oncol 32(3): 182-192, 2015. PMID: 25247487. DOI: 10.3109/ 08880018.2014.949941

10 Fiore A, Carraresi L, Morabito A, Polvani S, Fortunato A, Lastraioli E, Femia AP, De Lorenzo E, Caderni G and Arcangeli A: Characterization of hERG1 channel role in mouse colorectal carcinogenesis. Cancer Med 2(5): 583-594, 2013. PMID: 24403225. DOI: $10.1002 / \mathrm{cam} 4.72$

11 Glassmeier G, Hempel K, Wulfsen I, Bauer CK, Schumacher U and Schwarz JR: Inhibition of HERG1 K+ channel protein expression decreases cell proliferation of human small cell lung cancer cells. Pflugers Arch 463(2): 365-376, 2012. PMID: 22075718. DOI: $10.1007 / \mathrm{s} 00424-011-1045-\mathrm{z}$

12 Li H, Liu L, Guo T, Zhang J, Li X, Du W, Liu W, Chen X and Huang S: Expression and functional role of HERG1, K+ channels in leukemic cells and leukemic stem cells. J Huazhong Univ Sci Technolog Med Sci 27(3): 257-260, 2007. PMID: 17641836. DOI: 10.1007/s11596-007-0310-2

13 Masi A, Becchetti A, Restano-Cassulini R, Polvani S, Hofmann G, Buccoliero AM, Paglierani M, Pollo B, Taddei GL, Gallina P, Di Lorenzo N, Franceschetti S, Wanke E and Arcangeli A: hERG1 channels are overexpressed in glioblastoma multiforme and modulate VEGF secretion in glioblastoma cell lines. Br J Cancer 93(7): 781-792, 2005. PMID: 16175187. DOI: 10.1038/sj.bjc. 6602775

14 Shao XD, Wu KC, Guo XZ, Xie MJ, Zhang J and Fan DM: Expression and significance of HERG protein in gastric cancer.
Cancer Biol Ther 7(1): 45-50, 2008. PMID: 17938585 . DOI: 10.4161/cbt.7.1.5126

15 Suzuki T and Takimoto K: Selective expression of HERG and $\mathrm{Kv} 2$ channels influences proliferation of uterine cancer cells. Int J Oncol 25(1): 153-159, 2004. PMID: 15202000.

16 Jehle J, Schweizer PA, Katus HA and Thomas D: Novel roles for hERG $\mathrm{K}(+)$ channels in cell proliferation and apoptosis. Cell Death Dis 2: e193, 2011. PMID: 21850047. DOI: $10.1038 /$ cddis .2011 .77

17 Vandenberg JI, Perry MD, Perrin MJ, Mann SA, Ke Y and Hill AP: hERG $\mathrm{K}(+)$ channels: structure, function, and clinical significance. Physiol Rev 92(3): 1393-1478, 2012. PMID: 22988594. DOI: $10.1152 /$ physrev.00036.201118

18 He L and Hannon GJ: MicroRNAs: small RNAs with a big role in gene regulation. Nat Rev Genet 5(7): 522-531, 2004. PMID 15211354. DOI: $10.1038 / \mathrm{nrg} 1379$

19 Rupaimoole R and Slack FJ: MicroRNA therapeutics: towards a new era for the management of cancer and other diseases. Nat Rev Drug Discov 16(3): 203-222, 2017. PMID: 28209991. DOI: 10.1038/nrd.2016.246

20 Zhi D, Zhao X, Dong $M$ and Yan C: miR-493 inhibits proliferation and invasion in pancreatic cancer cells and inversely regulated hERG1 expression. Oncol Lett 14(6): 73987404, 2017. PMID: 29344180. DOI: 10.3892/ol.2017.7178

21 Feng J, Yu J, Pan X, Li Z, Chen Z, Zhang W, Wang B, Yang L, $\mathrm{Xu} \mathrm{H}$, Zhang $\mathrm{G}$ and $\mathrm{Xu} \mathrm{Z}$ : HERG1 functions as an oncogene in pancreatic cancer and is downregulated by miR-96. Oncotarget 5(14): 5832-5844, 2014. PMID: 25071021. DOI: 10.18632/ oncotarget 2200

22 Peng Y and Croce CM: The role of MicroRNAs in human cancer. Signal Transduct Target Ther 1: 15004, 2016. PMID: 29263891. DOI: $10.1038 /$ sigtrans.2015.4

23 Xie B, Ding Q, Han H and Wu D: miRCancer: a microRNAcancer association database constructed by text mining on literature. Bioinformatics 29(5): 638-644, 2013. PMID: 23325 619. DOI: $10.1093 /$ bioinformatics/btt014

24 Becker S: A historic and scientific review of breast cancer: The next global healthcare challenge. Int J Gynaecol Obstet 131: S3639, 2015. PMID: 26433503. DOI: 10.1016/j.ijgo.2015.03.015

25 Asher V, Sowter H, Shaw R, Bali A and Khan R: Eag and HERG potassium channels as novel therapeutic targets in cancer. World J Surg Oncol 8: 113, 2010. PMID: 21190577. DOI: 10.1186/14777819-8-113

26 Gong JH, Liu XJ, Shang BY, Chen SZ and Zhen YS: HERG K+ channel related chemosensitivity to sparfloxacin in colon cancer cells. Oncol Rep 23(6): 1747-1756, 2010. PMID: 20428834. DOI: $10.3892 /$ or_00000820

27 Shao XD, Wu KC, Hao ZM, Hong L, Zhang J and Fan DM: The potent inhibitory effects of cisapride, a specific blocker for human ether-a-go-go-related gene (HERG) channel, on gastric cancer cells. Cancer Biol Ther 4(3): 295-301, 2005. PMID: 15846098. DOI: $10.4161 / \mathrm{cbt} .4 .3 .1500$

28 Thomas D, Gut B, Karsai S, Wimmer AB, Wu K, WendtNordahl G, Zhang W, Kathofer S, Schoels W, Katus HA, Kiehn $\mathrm{J}$ and Karle CA: Inhibition of cloned HERG potassium channels by the antiestrogen tamoxifen. Naunyn Schmiedebergs Arch Pharmacol 368(1): 41-48, 2003. PMID: 12827215. DOI: 10.1007/s00210-003-0766-8

29 Christensen LL, Tobiasen H, Holm A, Schepeler T, Ostenfeld MS, Thorsen K, Rasmussen MH, Birkenkamp-Demtroeder K, 
Sieber OM, Gibbs P, Lubinski J, Lamy P, Laurberg S, Oster B, Hansen KQ, Hagemann-Madsen R, Byskov K, Orntoft TF and Andersen CL: MiRNA-362-3p induces cell cycle arrest through targeting of E2F1, USF2 and PTPN1 and is associated with recurrence of colorectal cancer. Int J Cancer 133(1): 67-78, 2013. PMID: 23280316. DOI: $10.1002 / \mathrm{ijc} .28010$

30 Kang H, Kim C, Lee H, Rho JG, Seo JW, Nam JW, Song WK, Nam SW, Kim W and Lee EK: Downregulation of microRNA362-3p and microRNA-329 promotes tumor progression in human breast cancer. Cell Death Differ 23(3): 484-495, 2016. PMID: 26337669. DOI: 10.1038/cdd.2015.116

31 Li M, Liu Q, Lei J, Wang X, Chen X and Ding Y: MiR-362-3p inhibits the proliferation and migration of vascular smooth muscle cells in atherosclerosis by targeting ADAMTS1. Biochem Biophys Res Commun 493(1): 270-276, 2017. PMID: 28890348. DOI: $10.1016 /$ j.bbrc.2017.09.031

32 Shen H, Li W, Tian Y, Xu P, Wang H, Zhang J and Li Y: Upregulation of miR-362-3p modulates proliferation and anchorage-independent growth by directly targeting Tob2 in hepatocellular carcinoma. J Cell Biochem 116(8): 1563-1573, 2015. PMID: 25649327. DOI: 10.1002/jcb.25110:

33 Wang D, Wang H, Li Y and Li Q: MiR-362-3p functions as a tumor suppressor through targeting MCM5 in cervical adenocarcinoma. Biosci Rep 38(3), 2018. PMID: 29871972. DOI: $10.1042 / \mathrm{bsr} 20180668$

34 Wang N, Feng Y, Xu J, Zou J, Chen M, He Y, Liu H, Xue M and $\mathrm{Gu}$ Y: miR-362-3p regulates cell proliferation, migration and invasion of trophoblastic cells under hypoxia through targeting Pax3. Biomed Pharmacother 99: 462-468, 2018. PMID: 29665647. DOI: $10.1016 /$ j.biopha.2018.01.089
35 Zhang QH, Yao YL, Wu XY, Wu JH, Gu T, Chen L, Gu JH, Liu $\mathrm{Y}$ and $\mathrm{Xu} \mathrm{L}$ : Anti-mir-362-3p inhibits migration and invasion of human gastric cancer cells by its target CD82. Dig Dis Sci 60(7): 1967-1976, 2015. PMID: 25652145. DOI: 10.1007/s10620-0153563-6

36 Zou X, Zhong J, Li J, Su Z, Chen Y, Deng W, Li Y, Lu S, Lin Y, Luo L, Li Z, Cai Z and Tang A: miR-362-3p targets nemolike kinase and functions as a tumor suppressor in renal cancer cells. Mol Med Rep 13(1): 994-1002, 2016. PMID: 26647877. DOI: $10.3892 / \mathrm{mmr} .2015 .4632$

37 Roy J, Vantol B, Cowley EA, Blay J and Linsdell P: Pharmacological separation of hEAG and hERG K+ channel function in the human mammary carcinoma cell line MCF-7. Oncol Rep 19(6): 1511-1516, 2008. PMID: 18497958.

38 Staudacher I, Jehle J, Staudacher K, Pledl HW, Lemke D, Schweizer PA, Becker R, Katus HA and Thomas D: HERG K+ channel-dependent apoptosis and cell cycle arrest in human glioblastoma cells. PLoS One 9(2): e88164, 2014. PMID: 24516604. DOI: 10.1371/journal.pone.0088164
Received August 2, 2019

Revised August 27, 2019

Accepted August 29, 2019 